\title{
Interannual correlations between sea surface temperature and concentration of chlorophyll pigment off Punta Eugenia, Baja California, during different remote forcing conditions
}

\author{
H. Herrera-Cervantes et al.
}

Correspondence to: H. Herrera-Cervantes (hherrera@cicese.mx) 

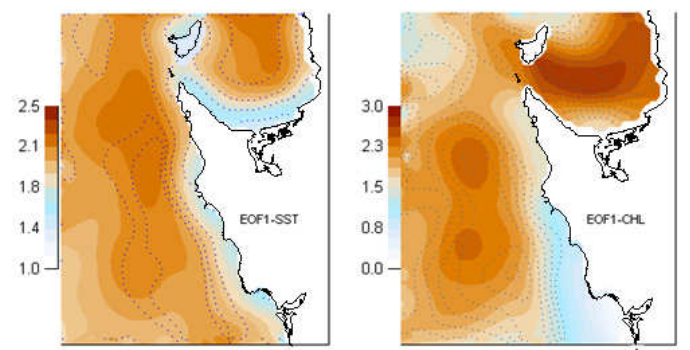

Figure 1. Spatial patterns for mode 1 of the individual EOF analyses. SST (left), Chlorophyll a (right, (with the sign reversed). Overlaid on the spatial component maps are contours (dotted) of the homogeneous correlation.

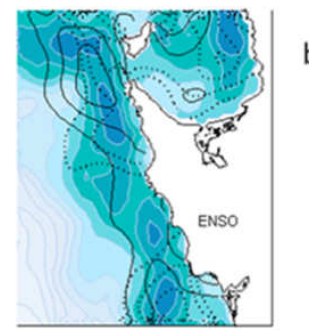

b

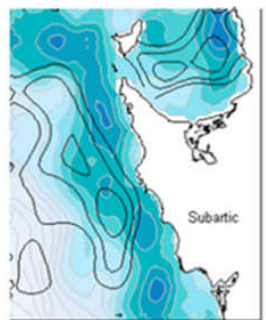

Figure 2. a) HCs for the ENSO period (dotted an solid contours) overlaid on the correlation map (a) present spatial trends of SST and Chl-a in a broad band along the coast and (b) HGs patterns of subartic water (solid contours) partially coincide with the band of high correlations values.
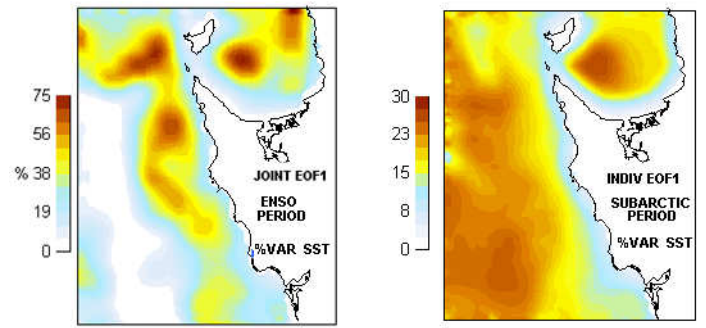

Figure 3. Spatial distribution of SST variance in both ENSO and SUBARCTIC periods. These patterns are similar to Joint and individual EOF 1 patterns. 


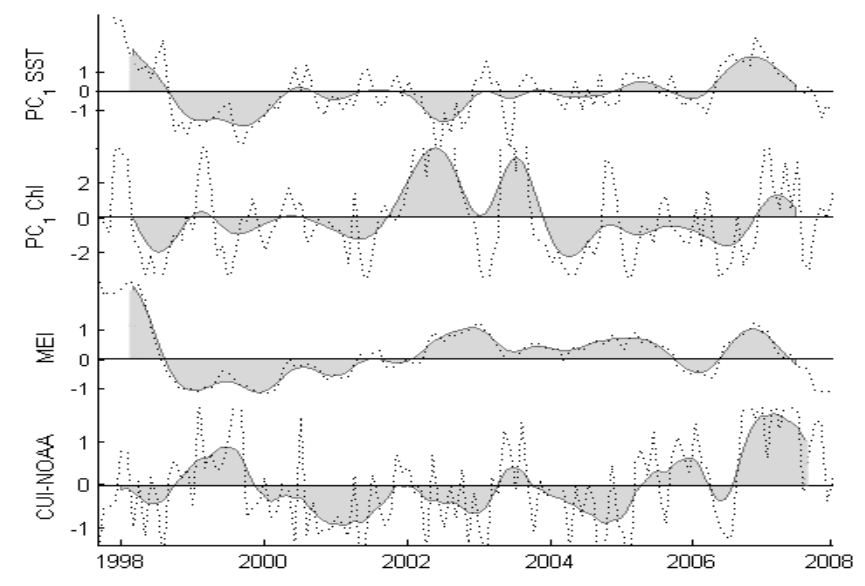

Figure 4, Time-series data (shaded) indicate the temporal evolution of both SST AND Chl-a EOF $_{1}$ alongside the MEI and monthly CUI anomalies, all smoothed by a double five-term running mean. Dotted lines indicate the original data.

Table 1. Correlations for original monthly time series $(N=124)$

\begin{tabular}{c|cccccc} 
& $N$ & $R$ & tau & $N_{\text {eff }}$ & $\begin{array}{c}t- \\
\text { student }\end{array}$ & $P_{\text {val }}$ \\
\hline $\mathrm{PC}_{1 \text { SST }-\mathrm{MEI}}$ & 124 & 0.66 & 3.0 & 42 & 5.5 & $P<10^{-6}$ \\
$\mathrm{PC}_{1 \mathrm{CHL}}-\mathrm{MEI}$ & 124 & -0.10 & 1.7 & 74 & 0.4 & $P<10^{-1}$ \\
$\mathrm{PC}_{1}$ SST $-\mathrm{CUI}$ & 124 & -0.13 & 1.7 & 70 & 1.1 & $P<10^{-1}$ \\
$\mathrm{PC}_{1} \mathrm{CHL}-\mathrm{CUI}$ & 124 & -0.30 & 1.2 & 100 & 3.0 & $P<10^{-3}$ \\
$\mathrm{PC}_{1 \text { SST }}-\mathrm{CP}_{1 \mathrm{CHL}}$ & 124 & 0.20 & 1.4 & 85 & 1.4 & $P<10^{-2}$
\end{tabular}

Table 2. Correlations for the period ENSO, $\mathrm{N}=28$ months.

\begin{tabular}{l|lcccll} 
& $N$ & $R$ & tau & Neff & $t$-student & $P$ val \\
\hline $\mathrm{PC}_{1 \text { SST }- \text { Mei }}$ & 28 & 0.87 & 4.0 & 7 & 4.0 & $\mathrm{P}<10^{-2}$ \\
$\mathrm{PC}_{1}$ CHL - Mei & 28 & -0.10 & 1.0 & 25 & 0.5 & $\mathrm{P}<10^{-1}$ \\
$\mathrm{PC}_{1 \text { SST }}$-CUI & 28 & -0.54 & 1.5 & 17 & 2.5 & $\mathrm{P}<10^{-2}$ \\
$\mathrm{CP}_{1}$ CHL - CUI & 28 & -0.24 & 1.0 & 27 & 1.2 & $\mathrm{P}<10^{-1}$
\end{tabular}

Table 3. Correlations for the period SUBARCTIC, $\mathrm{N}=24$ months.

\begin{tabular}{l|lrrlll} 
& $N$ & $R$ & tau & Neff & $t$-student & Pval \\
\hline $\mathrm{PC}_{1 \text { SST }}$-Mei & 28 & -0.10 & 1.3 & 17 & 1.3 & $\mathrm{P}<10^{-1}$ \\
$\mathrm{PC}_{1 \text { CHL }}$-Mei & 28 & 0.40 & 1.5 & 15 & 1.5 & $\mathrm{P}<10^{-1}$ \\
$\mathrm{PC}_{1 \text { SST }}$-CUI & 28 & -0.19 & 1.1 & 21 & 1.1 & $\mathrm{P}<10^{-1}$ \\
$\mathrm{CP}_{1 \text { CHL }}$-CUI & 28 & -0.62 & 1.2 & 19 & 1.2 & $\mathrm{P}<10^{-3}$
\end{tabular}

